\title{
Experimental investigation into segregating granular flows down chutes
}

\author{
Sébastien Wiederseiner, ${ }^{1}$ Nicolas Andreini, ${ }^{1}$ Gaël Épely-Chauvin, ${ }^{1}$ Gaudenz Moser, ${ }^{1}$ \\ Mathieu Monnereau, ${ }^{2}$ J. M. N. T. Gray, ${ }^{3}$ and Christophe Ancey ${ }^{1}$ \\ ${ }^{1}$ École Polytechnique Fédérale de Lausanne, Écublens, 1015 Lausanne, Switzerland \\ ${ }^{2}$ Département Hydraulique et Mécaniques des Fluides, ENSEEIHT, BP 7122, \\ 31071 Toulouse Cedex 7, France \\ ${ }^{3}$ School of Mathematics and Manchester Centre for Nonlinear Dynamics, University of Manchester, \\ Manchester M13 9PL, United Kingdom
}

(Received 25 June 2010; accepted 20 December 2010; published online 6 January 2011)

\begin{abstract}
We experimentally investigated how a binary granular mixture made up of spherical glass beads (size ratio of 2) behaved when flowing down a chute. Initially, the mixture was normally graded, with all the small particles on top of the coarse grains. Segregation led to a grading inversion, in which the smallest particles percolated to the bottom of the flow, while the largest rose toward the top. Because of diffusive remixing, there was no sharp separation between the small-particle and large-particle layers, but a continuous transition. Processing images taken at the sidewall, we were able to measure the evolution of the concentration and velocity profiles. These experimental profiles were used to test a recent theory developed by Gray and Chugunov [J. Fluid Mech. 569, 365 (2006)], who derived a nonlinear advection diffusion equation that describes segregation and remixing in dense granular flows of binary mixtures. We found that this theory was able to provide a consistent description of the segregation/remixing process under steady uniform flow conditions. To obtain the correct depth-averaged concentration far downstream, it was very important to use an accurate approximation to the downstream velocity profile through the avalanche depth. The $S$-shaped concentration profile in the far field provided a useful way of determining what we refer to as the Péclet number for segregation, a dimensionless number that quantifies how large the segregation is compared to diffusive remixing. While the theory was able to closely match the final fully developed concentration profile far downstream, there were some discrepancies in the inversion region (i.e., the region in which the mixing occurs). The reasons for this are not clear. The difficulty to set up the experiment with both well controlled initial conditions and a steady uniform bulk flow field is one of the most plausible explanations. Another interesting lead is that the flux of segregating particles, which was assumed to be a quadratic function of the concentration in small beads, takes a more complicated form. (c) 2011 American Institute of Physics.
\end{abstract}

[doi:10.1063/1.3536658]

\section{INTRODUCTION}

Segregation processes are ubiquitous in granular flows involving a wide range of particle sizes. Many flows in nature and industry give the opportunity to observe the effects of segregation, e.g., granular deposits of wet-snow avalanches and rockfalls, ${ }^{1,2}$ dosing from vending machine canisters. ${ }^{3}$ Among the numerous processes that cause segregation, kinetic sieving and squeeze expulsion are likely to be the most efficient in dense dry granular flows down chutes: ${ }^{4}$ velocity shear and dilatancy act together as a random fluctuating sieve that allows the finer particles to percolate to the bottom under the action of gravity, while squeezing larger particles upward. Understanding size segregation is of paramount importance to understanding the dynamics of granular flows since it can have a feedback on the bulk flow, e.g., by reducing bottom friction during the course of motion., ${ }^{5,6}$

Segregation has been mostly studied in rotating drums and cells ${ }^{7,8}$ (e.g., long horizontal or thin rotating drums) and shakers, ${ }^{9}$ for which a large body of experimental work exists. On rare occasions, segregation has been studied using chutes. Savage and $\operatorname{Lun}^{4}$ measured the development of con- centration profiles of granular flows down an inclined flume. A series of splitter plates were located at the downstream end of the flume, which made it possible to collect particles in separate bins. By measuring the proportion of small particles in each bin, the authors obtained a histogram of concentrations, which gave an idea of the concentration profile. The device had the advantage of measuring width-averaged values of the concentration, thus minimizing the influence of sidewalls. However, it also had the disadvantage of disturbing the flow (by modifying the flow depth) and yielding poor-resolution data. Dolgunin and Ukolov ${ }^{10}$ used an experimental setup similar to Savage and $\mathrm{Lun}^{4}$ except that they did not use splitter plates. Instead, they let the particles flow into bins located under the downstream end of the chute. The particles fell into separate bins depending on their velocity and position as they left the chute. While this technique did not disturb the flow in the chute, it suffered from several deficiencies. In particular, particle deposition into bins was affected by air resistance and like Savage and Lun's experiments, binning provided poor-resolution data. More recently, Golick and Daniels ${ }^{11}$ and May et al. ${ }^{12}$ used an annular shear 
cell to study how remixing and segregation were influenced by the normal stress (confining pressure). Velocity profiles were measured at the sidewall using a high-speed camera and segregation and mixing effects were estimated indirectly by measuring the expansion and compaction of the aggregate through the rise and fall of the top plate.

Segregation in dense granular flows has been investigated theoretically using different approaches including information-entropy theory, ${ }^{4}$ statistical mechanics, ${ }^{13}$ and binary-mixture theory. ${ }^{14}$ For dense granular flows, the last theoretical approach is interesting in that it provides a relatively simple description of segregation-remixing in the form of a nonlinear advection diffusion equation for each solid component. This equation (outlined below) does not depend on a particular form of governing equations for the bulk and it is therefore compatible with most existing granular-flow models. For dense granular flows, approximate governing equations can be derived within the framework of flow-depth averaged equations, ${ }^{15-17}$ various forms of these governing equations have been proposed, the main difference lying in the choice of constitutive equation.

The objective of this paper is to test Gray and Chugunov's theory ${ }^{14}$ for segregation and diffusive remixing in granular avalanches against experimental data. The experiment consists of a steady uniform flow of a binary granular mixture, in which there is an inversion in the small-particle concentration as the grains readjust into a stable configuration. Initially, as the grains flow out of a hopper, the bulk flow is normally graded, with all the small particles on top of the coarse grains. As a result of segregation, the smallest particles percolate to the bottom, while the largest rise to the top, producing an inversely graded flow. Diffusive remixing ensures that there is no sharp separation between the smallparticle and large-particle layers, but a continuous transition.

The paper is organized as follows. In Sec. II, we outline the theoretical developments that lead to the nonlinear advection diffusion equation for segregation and diffusive remixing. Section III deals with the experimental setup. In Sec. IV, we present some of our experimental data and compare them with theoretical predictions. Section V summarizes and discusses our main findings.

\section{THEORY}

Consider an avalanche of large and small particles of the same density that is flowing down a slope inclined at an angle $\zeta$ to the horizontal (see Fig. 1). Following Gray and Chugunov, ${ }^{14}$ we define a Cartesian coordinate system in which the $x$-axis points down the chute, the $y$-axis points across the slope, and the $z$-axis is in the direction of the upward pointing normal. The bulk velocity $\mathbf{u}$ has components $u, v$, and $w$ in each of these directions, respectively. During the flow the avalanche is sheared and dilates sufficiently for the small particles to percolate down into gaps that open up beneath them, under gravity, and there is a return flow of large particles toward the free surface as they are levered up by squeeze expulsion. ${ }^{4}$ The solids volume fraction is assumed to equilibrate itself so that it is constant and uniform throughout the flow. This allows the free space

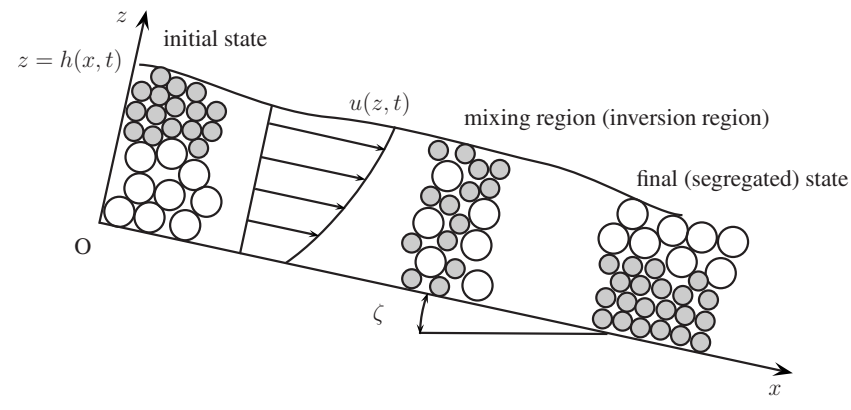

FIG. 1. Sketch of a granular flow down a chute. At the flume entrance, the binary mixture is normally graded (with all the fine particles on top the coarse particles), but as the particles flow down the chute, the large grains gradually rise to the surface while the small ones percolate down to the base. This creates an inversely graded layer far downstream. The free surface lies at $z=h(x, t)$. The arrows indicate the downstream velocity profile $u(z, t)$ through the layer.

to be subsumed into the bulk density $\rho$ without having to explicitly consider the interstitial fluid. ${ }^{18}$ The volume fractions of large and small particles, $\phi^{l}$ and $\phi^{s}$, can therefore be defined per unit mixture volume. The sum of the volume fractions is necessarily equal to unity,

$$
\phi^{l}+\phi^{s}=1 .
$$

The assumption of spatial uniformity of the solids volume fraction is a reasonable first approximation. In real flows a mixture of large and small grains has a lower voids fraction than in either of the pure phases, since the smaller grains can fit in the gaps between the large ones. This enhanced packing gives rise to the top plate motion in Golick and Daniels ${ }^{11}$ experiment. The expansion and contraction of the flow as it segregates is similar in magnitude to the dilation that occurs as the grains are mobilized from the rest state. In Golick and Daniels' ${ }^{11}$ experiment this accounted for a $2.5 \%$ change in the flow height. These changes may affect the segregation rates, but very little is known about them, especially in sheared systems, ${ }^{12}$ and they are neglected in the current model.

In common with most avalanche models ${ }^{15,16}$ it is assumed that the acceleration terms and the deviatoric stress gradients are small compared to the pressure gradient in the normal direction. This allows the normal component of the momentum balance to be integrated through the avalanche depth to show that the pressure is lithostatic,

$$
p=\rho g(h-z) \cos \zeta,
$$

where $g$ is the constant of gravitational acceleration and the height $h=h(x, y, t)$ is a function of $x, y$, and time $t$. Mixture theory ${ }^{19,20}$ defines overlapping partial and intrinsic pressures, $p^{\nu}$ and $p^{\nu *}$, which are defined per unit mixture volume and per unit volume of constituent $\nu$, respectively. These are usually related by a linear volume fraction scaling, i.e., $p^{\nu}=\phi^{\nu} p^{\nu *}$. However, Gray and Thornton ${ }^{21}$ argued that as the small particles percolate down through the matrix of large grains, they support proportionately less of the overburden pressure and the large grains must necessarily support more of the load. This led them to introduce a nonlinear partial intrinsic pressure scaling of the form 


$$
p^{\nu}=f^{\nu} p
$$

where the factor $f^{\nu}$ could deviate away from $\phi^{\nu}$. Guided by the fact that $f^{l}+f^{s}=1$, in order that the partial pressures sum to the lithostatic pressure, and that $f^{\nu}$ must be equal to unity when constituent $\nu=l, s$, is in a pure phase, Gray and Thornton ${ }^{21}$ postulated a very simple quadratic form for the perturbations away from $\phi^{\nu}$,

$$
f^{l}=\phi^{l}+b \phi^{s} \phi^{l} \quad \text { and } f^{s}=\phi^{s}-b \phi^{s} \phi^{l},
$$

where $b$ is the magnitude of the perturbation. The forces between the large and small particles are determined by the interaction drag terms in the constituent momentum balance equations. ${ }^{19}$ Gray and Chugunov ${ }^{14}$ assumed that they consisted of a term to ensure the particles were driven by intrinsic rather than partial pressure gradients, a linear velocity dependent drag and a concentration dependent remixing force that drove particles to regions of lower concentration. When these are substituted into the normal component of the constituent momentum balances the normal velocities of the large and small particles are

$$
\begin{aligned}
& w^{l}=w+q \phi^{s}-D \frac{\partial}{\partial z} \ln \phi^{l}, \\
& w^{s}=w-q \phi^{l}-D \frac{\partial}{\partial z} \ln \phi^{s},
\end{aligned}
$$

where $q$ is the maximum percolation rate of the grains and $D$ is the diffusivity, which controls the strength of the remixing. In the absence of diffusive remixing, $D=0$, we observe that large particles rise until there are $100 \%$ coarse grains and that the small particles percolate down until there are $100 \%$ small grains to create inversely graded sharply segregated layers. Diffusive remixing tries to smooth out sharp concentration gradients in all directions and may either aid or oppose the segregation. We assume that the percolation and diffusive remixing velocities are of the same order of magnitude as typical normal velocities of the bulk flow. Since the avalanche is very long and thin, these are much smaller than typical downstream and cross slope velocities. We therefore assume that the constituent velocities in the down and cross slope directions are equal to the bulk flow components,

$$
u^{\nu}=u \quad \text { and } \quad v^{\nu}=v \text {, }
$$

with $\nu=l, s$. As a result, segregation and diffusive remixing in the downstream or cross-stream directions is neglected. When the velocity components (6) and (7) and the largeparticle concentration $\phi^{l}=1-\phi^{s}$ are substituted into the small-particle mass balance equation, ${ }^{19}$ they yield the segregation-remixing equation

$$
\frac{\partial \phi}{\partial t}+\operatorname{div}(\phi \mathbf{u})-\frac{\partial}{\partial z}[q \phi(1-\phi)]=\frac{\partial}{\partial z}\left(D \frac{\partial \phi}{\partial z}\right),
$$

where we have dropped the superscript $s$ on the smallparticle concentration for simplicity, i.e., here and throughout the rest of the paper $\phi \equiv \phi^{s}$.

The first term on the left-hand side of Eq. (8) is the time rate of change of the small-particle concentration and the second term is due to advection by the bulk velocity field $\mathbf{u}=(u, v, w)$. The third nonlinear term accounts for segregation, while the right-hand side introduces the diffusive effects of remixing. Mathematically, this equation is a second-order parabolic equation when $D>0$; for $D=0$, it reduces to a first order scalar conservation law, which is consistent with the equation derived by Savage and $\mathrm{Lun}^{4}$ using informationentropy theory. Owing to its hyperbolic nature, Eq. (8) with $D=0$ may form shocks, i.e., waves across which the smallparticle concentration experiences a jump. When $D>0$, diffusive remixing smears out the shock wave, replacing it by a smooth transition in the small-particle concentration.

At that level, we have no specific knowledge of how $q$ and $D$ vary with particle size, shear rate, etc. Mixture theory is of little help to elucidate this point. Using their information-entropy approach, Savage and Lun ${ }^{4}$ gave more information on $q$ by showing that it is related to the probability that a small particle falls into the void space between large particles. In the $\phi \rightarrow 0$ limit, they found that the percolation $q$ is proportional to the shear rate $\dot{\gamma}=\partial u / \partial z$ and the particle-diameter ratio. This behavior is consistent with the measurements taken by Bridgwater et al. ${ }^{22}$ In other settings ${ }^{23}$ (for density-driven segregation), the segregation flux is found to be dependent on many parameters, including the particle diameter. For the sake of simplicity (and making allowance for our narrow range of experimental conditions), we assume that $q$ and $D$ are constant constitutive parameters. This assumption does not cause much trouble in Gray and Chugunov's theory since these authors considered linear velocity profiles (i.e., $\dot{\gamma}$ is constant across the flow depth), but it may lead to difficulties here since we do not consider linear profiles.

Assuming $q$ and $D$ constant makes it possible to work out exact analytical solutions to Eq. (8) for certain initial boundary-value problems. For instance, when the bulk transport term is zero, Eq. (8) is closely related to the Burgers equation and therefore can be solved using the Cole-Hopf transformation. ${ }^{14}$ When diffusive remixing has negligible effect, exact solutions can be derived using the method of characteristics. $^{18,21,24-26}$

If the avalanche has constant uniform thickness and the particle size distribution is also steady and laterally uniform in the down and cross slope directions, Eq. (8) reduces to

$$
-\frac{d}{d z}[q \phi(1-\phi)]=\frac{d}{d z}\left(D \frac{d \phi}{d z}\right)
$$

and is subject to no-flux boundary conditions,

$$
q \phi(1-\phi)+D \frac{d \phi}{d z}=0 \text { at } z=0 \text { and } h .
$$

Integrating Eq. (9) with respect to $z$ and using Eq. (10) at either of the boundaries yields a first order ordinary differential equation, which can be integrated ${ }^{14}$ to show that steady laterally uniform concentration of small grains is 


$$
\phi_{\text {uniform }}(z)=\frac{1}{1+A \exp (q z / D)},
$$

where $A$ is an integration constant. The boundary conditions (10) are not sufficient to determine $A$ and a further criterion must be imposed. Assuming that the mean concentration of small particles,

$$
\bar{\phi}=\frac{1}{h} \int_{0}^{h} \phi(z) d z
$$

is fixed, we can integrate Eq. (11) to determine

$$
A=\frac{\exp (-q h \bar{\phi} / D)-\exp (-q h / D)}{1-\exp (-q h \bar{\phi} / D)} .
$$

Note that the solution in Eq. (11) is independent of the actual velocity profile and depends on the percolation rate and diffusivity through the ratio $q / D$. In this equation, we can also introduce a dimensionless ratio $\mathrm{Pe}=q h / D$, which can be referred to as the Péclet number for segregation and quantifies how large the segregation effects are compared to the diffusive effects (remixing); in this ratio, the advection velocity is the percolating velocity $q$ and the characteristic length is the flow-depth $h$. As will be shown later, the steady laterally uniform solution has an $S$-shaped form for finite values of the Péclet number. For large Péclet numbers, there is a rapid transition from small- to large-particle concentration with increasing height with states approaching the pure phases at the top and the bottom of the flow. For small Péclet numbers, the grading appears linear with depth while the surface and basal values may be far from the pure states. For $\mathrm{Pe} \rightarrow \infty$ (pure segregation), the particles separate out to form two distinct inversely graded layers with all the large particles on top of all the fines and a sharp concentration jump at $z=\bar{\phi} h$. At the opposite extreme, for $\mathrm{Pe} \rightarrow 0$ (no segregation), the small particles are uniformly distributed with concentration $\phi(z)=\bar{\phi}$ throughout the depth of the flow. Later, Eq. (11) will be used to estimate the values of $q / D$.

In this paper we assume that the cross stream and normal velocities, $v$ and $w$, are equal to zero and we solve Eq. (8) for the steady-state solution in a steady uniform thickness flow using a Galerkin method described by Skeel and Berzins ${ }^{27}$ and implemented in MATLAB through the built-in function pdepe. The no-flux condition, Eq. (10), is applied at the surface and base of the flow and at the inflow the initial concentration is unstably stratified,

$$
\phi(0, z)= \begin{cases}1, & h_{1} \leq z \leq h=h_{1}+h_{2} \\ 0, & 0 \leq z \leq h_{1}\end{cases}
$$

with a layer of small particles of depth $h_{2}$ on top of a layer of large particles of depth $h_{1}$. Kinetic sieving and squeeze expulsion drive a readjustment of the particles into a stable configuration, in which, sufficiently far downstream, the solution approaches the steady laterally uniform solution in Eq. (11).

The laterally uniform solution (11) depends on the mean concentration $\bar{\phi}$. In general, $\bar{\phi}(x)$ is not uniform, but evolves from its inflow value to the steady uniform value far down- stream. To show this, we use Leibniz' integral theorem to integrate Eq. (8) through the flow depth ${ }^{28}$ assuming $v=0$; applying both the kinematic ${ }^{16}$ and no-flux [Eq. (10)] boundary conditions yields the depth-averaged segregation equation

$$
\frac{\partial}{\partial t}(h \bar{\phi})+\frac{\partial}{\partial x}(h \bar{\phi} u)=0,
$$

where the depth-averaged small-particle flux

$$
\overline{\phi u}=\frac{1}{h} \int_{0}^{h} \phi(x, z) u(x, z) d z \text {. }
$$

For steady-state concentration distributions, Eq. (15) implies that the total downstream flux of small particles is conserved, i.e.,

$$
h \overline{\phi u}=\text { const. }
$$

The conservation property implies that the depth-averaged concentration $\bar{\phi}$, in the exact solution, given by Eqs. (11) and (13), can be directly related to the inflow condition, Eq. (14). The depth-averaged conservation of total mass, when $v=0$, satisfies the continuity equation ${ }^{15}$

$$
\frac{\partial h}{\partial t}+\frac{\partial}{\partial x}(h \bar{u})=0
$$

which implies that for a steady flow,

$$
h \bar{u}=\text { const. }
$$

In a steady uniform thickness flow, the depth-averaged downstream velocity is therefore also independent of $x$. If either $\phi$ or $u$ is independent of $z$, then $\bar{\phi} u=\bar{\phi} \bar{u}$ and the conserved quantities (17) and (19) imply that $\bar{\phi}$ is uniform in a steady uniform thickness flow. In general, $\bar{\phi} \bar{u} \neq \bar{\phi} \bar{u}$ and the depth-averaged concentration of small particles $\bar{\phi}$ will evolve with the downstream coordinate $x$ as they percolate down into slower moving regions of the flow. Note that the depth-averaged downstream flux of small particles in Eq. (17) can be related to the total flow rate of small particles $Q_{\text {small }}$ (used later) by $Q_{\text {small }}=\rho W h \overline{\phi u}$, where $W$ is the flume width and $\rho$ is the constant bulk density.

\section{EXPERIMENTAL FACILITY AND PROCEDURES}

\section{A. Experimental setup and material}

Experiments were conducted in a rough-bottomed flume with glass sidewalls. The flume was $3 \mathrm{~m}$ long and $2 \mathrm{~cm}$ wide; the small width was desirable to avoid three-dimensional effects in the segregation patterns, but it produced sidewall friction that affected flow features. ${ }^{29}$ The flume laid on a $4 \mathrm{~m}$ long aluminum plate, which was supported by a frame made of profiled aluminum beams to ensure rigidity. It could be inclined from $0^{\circ}$ to $45^{\circ}$, but here all the experimental data reported were obtained with a slope $\zeta=29^{\circ}$. Its position was accurately controlled using a digital inclinometer with a precision of $0.1^{\circ}$. The flume base was roughened by inserting a double-sided adhesive tape, over which beads were glued. Filming the bed particles from the sidewall, we did not ob- 
TABLE I. Inflow flow-depth $h_{1}$ and $h_{2}$ in millimeters (see Fig. 2); duration of the experiment in seconds; mass flow rates in large and small particles, $Q_{\text {large }}$ and $Q_{\text {small }}$, respectively, in $\mathrm{g} / \mathrm{s}$; flow-depth $h$ (in millimeters) at different positions (in centimeters). All experiments were conducted at $\zeta=29^{\circ}$.

\begin{tabular}{|c|c|c|c|c|c|c|c|c|c|c|c|}
\hline \multirow[b]{2}{*}{ Run } & \multirow[b]{2}{*}{$h_{1}$} & \multirow[b]{2}{*}{$h_{2}$} & \multirow[b]{2}{*}{$t_{\text {expt }}$} & \multirow[b]{2}{*}{$Q_{\text {large }}$} & \multirow[b]{2}{*}{$Q_{\text {small }}$} & \multicolumn{6}{|c|}{$h$} \\
\hline & & & & & & $x=0$ & $x=3.5$ & $x=50$ & $x=100$ & $x=180$ & $x=260$ \\
\hline 1 & 25.4 & 6.3 & 71.5 & 55.6 & 22.3 & 31.7 & 24.0 & 24.0 & 21.5 & 20.5 & 17.0 \\
\hline 2 & 25.4 & 8.3 & 94.5 & 47.5 & 49.0 & 33.7 & 23.0 & 26.5 & 25.0 & 21.5 & 17.0 \\
\hline 3 & 25.4 & 10.3 & 85.6 & 47.0 & 60.9 & 35.7 & 23.5 & 26.0 & 25.0 & 22.5 & 17.5 \\
\hline 4 & 25.4 & 12.3 & 86.9 & 41.8 & 75.8 & 37.7 & 24.5 & 25.0 & 25.0 & 23.5 & 18.0 \\
\hline 5 & 25.4 & 14.3 & 90.2 & 40.8 & 92.9 & 39.7 & 20.5 & 24.0 & 26.0 & 26.5 & 21.0 \\
\hline 6 & 31.7 & 6.0 & 66.9 & 58.8 & 15.1 & 37.7 & 26.5 & 23.0 & 20.0 & 19.5 & 17.0 \\
\hline 7 & 31.7 & 8.0 & 63.5 & 54.5 & 26.3 & 39.7 & 27.5 & 25.0 & 22.0 & 20.0 & 17.0 \\
\hline 8 & 31.7 & 10.0 & 66.5 & 49.9 & 38.5 & 41.7 & 28.0 & 26.0 & 25.0 & 21.5 & 17.5 \\
\hline 9 & 31.7 & 12.0 & 64.3 & 47.2 & 57.5 & 43.7 & 29.0 & 27.0 & 28.0 & 25.0 & 19.0 \\
\hline 10 & 31.7 & 14.0 & 62.1 & 40.4 & 72.6 & 45.7 & 30.0 & 31.0 & 31.5 & 29.0 & 21.0 \\
\hline 11 & 35.1 & 6.0 & 69.6 & 53.3 & 21.6 & 41.1 & 28.0 & 27.5 & 22.0 & 20.5 & 16.5 \\
\hline 12 & 35.1 & 8.0 & 63.6 & 47.6 & 34.4 & 43.1 & 28.0 & 27.5 & 28.0 & 27.5 & 17.0 \\
\hline 13 & 35.1 & 10.0 & 67.3 & 43.8 & 50.3 & 45.1 & 28.5 & 28.0 & 28.5 & 28.5 & 19.0 \\
\hline 14 & 35.1 & 12.0 & 66.7 & 40.8 & 67.2 & 47.1 & 30.5 & 31.0 & 30.5 & 26.5 & 19.5 \\
\hline 15 & 35.1 & 14.0 & 64.3 & 34.2 & 84.7 & 49.1 & 34.0 & 32.0 & 31.0 & 27.0 & 20.0 \\
\hline 16 & 39.9 & 6.0 & 63.1 & 55.1 & 18.7 & 45.9 & 37.0 & 30.5 & 23.5 & 18.5 & 16.0 \\
\hline 17 & 39.9 & 8.0 & 65.9 & 50.3 & 29.2 & 47.9 & 38.0 & 34.0 & 31.0 & 23.0 & 17.0 \\
\hline 18 & 39.9 & 10.0 & 62.6 & 47.1 & 41.2 & 49.9 & 39.0 & 35.0 & 34.5 & 28.0 & 18.0 \\
\hline 20 & 39.9 & 12.0 & 64.7 & 42.9 & 52.0 & 51.9 & 39.0 & 35.5 & 35.0 & 29.5 & 20.5 \\
\hline 21 & 39.9 & 14.0 & 66.5 & 37.2 & 65.2 & 53.9 & 39.0 & 35.5 & 33.5 & 28.0 & 20.0 \\
\hline
\end{tabular}

serve any particle dislodged from the tape, but we could not ensure that this never happened. Tests with different roughness sizes were conducted and we finally selected $1 \mathrm{~mm}$ particles for the roughness because they allowed us to obtain uniform depth flows over a wider range of flow rates.

We used binary mixtures of small beads (ranging from 0.75 to $1 \mathrm{~mm}$ in diameter) and large beads $(2 \mathrm{~mm}$ in diameter, with a narrow distribution around this value). For both sizes, the density was $2500 \mathrm{~kg} / \mathrm{m}^{3}$. Small beads were transparent, while the larger ones were colored in black; this choice produced the best contrast for our images. To avoid electrostatic effects resulting from particle-particle and particle-sidewall friction, we grounded all metallic pieces (sieve, hopper, and frame). After each run, the particles were collected in a bin placed below the flume outlet; then they were poured in a cylindrical copper duct to remove electric charges as much as possible. Particles were also sieved and reused for the next experiment. Experiments were run under well controlled conditions (50\% humidity, $25{ }^{\circ} \mathrm{C}$ temperature). The flow rates of small particles ranged from 20 to $100 \mathrm{~g} \mathrm{~s}^{-1}$, while the flow rates of large particles were in the 35-60 $\mathrm{g} \mathrm{s}^{-1}$ range (see Table I).

Initially, each bead class was placed in a separate reservoir attached to the flume inlet. As sketched in Fig. 2, the reservoirs were separated by a splitter plate, whose inclination partially controlled flow rates of both the large and small beads; they were supplied with beads by two cylindrical hoppers. There were numerous constraints that made the design of the splitter and reservoirs difficult. First, the velocity profile had to be as continuous as possible, which implied that the splitter plate had to be inclined at a shallow slope (i.e., parallel to the flume base) in order to avoid disturbances. Second, the velocity mismatch between small and large beads at their interface needed to be as low as possible. Third, the position and inclination of the splitter plate had to vary in order to adjust the respective inflow rate of large and small particles. After much trial and error, a deflecting plate was added in the lower reservoir to help the large particles to follow streamlines parallel to the base; the walls of the upper reservoir containing small particles were made rougher to reduce their velocity.

\section{B. Image processing}

In order to investigate how small and large particles segregate when flowing down the flume under steady flow conditions, small beads were injected from above while large particles crept along the flume base. The small particles rapidly percolated to the bottom, while the large ones drifted to the top of the flow. In this setting, the temporal vertical seg-

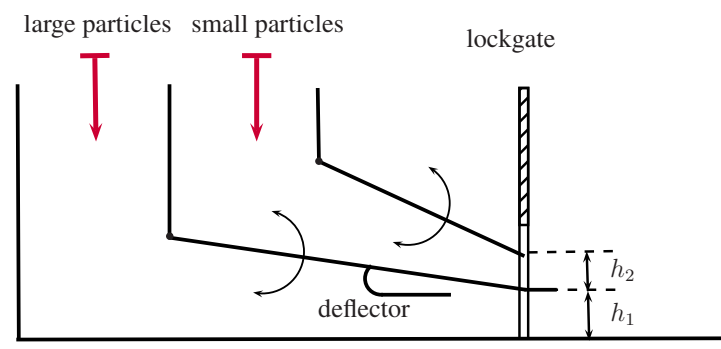

FIG. 2. (Color online) Sketch of the feeding system. The arrowed arcs indicate that the plates can be tilted. 


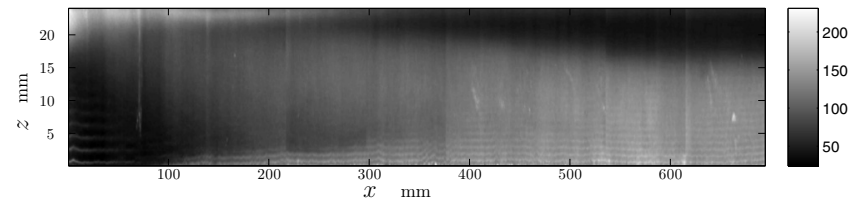

FIG. 3. Raw image of the segregation process obtained by averaging 2000 images at ten downstream locations and stitching them together. Darker regions correspond to higher concentrations of large grains and lighter regions to higher concentration of fines (the sidebar on the right is the gray level index, which ranges from 0 to 255 since the image was 8 bit encoded). The flow is from left to right with the inflow at $x=0$. Note that the height to length ratio is 0.0343 , so that horizontal gradients are much less than they might appear.

regation process was visible through the downstream variation in the small-particle concentration $\phi(x, z)$. This segregation process was filmed by a high-speed camera (Basler piA640, $648 \times 488$ pixels) running at $160 \mathrm{fps}$, which was mounted on a mobile frame. The camera equipped with a $12.5 \mathrm{~mm}$ lens (Fujinon HF12.5SA-1) was able to film a $7 \mathrm{~cm}$ wide field, which was free of vignetting effects. To increase the length of the observing window up to $70 \mathrm{~cm}$, we reproduced the same experiment ten times and for each run, we shifted the camera by $7 \mathrm{~cm}$ along a glider and took a series of 2000 images. Then, we averaged these 2000 images to obtain a mean-value image. Finally, we stitched ten mean-value images to obtain a panoramic view of the flume, as shown in Fig. 3.

Two halogen lamps were linked to the camera. They lit the same pane as the one filmed by the camera, which made it possible to measure the velocity and concentration profiles at the sidewall with the same light conditions even when the camera was displaced. Note that there are some horizontal streaks in Fig. 3, in particular in the bottom left corner, which may lead to the erroneous conclusion that there were local sharp gradients in the particle concentration near the flume bottom. These light streaks originated from the glares produced on spherical particles by the halogen lamps; the local brightness increase due to these glares induced an apparent increase in the small-particle concentration. Moreover, they also neatly delineated slowly moving regions of almost purely large and purely small particles, which developed near the base of the flow.

To measure the concentration profiles at the sidewall, we needed to map the gray levels ( 8 bit encoding, i.e., 256 gray levels) of our images to the concentration of small particles. To that end, we prepared different samples by varying the proportions of small and large beads. Each sample was gently poured in a cylindrical transparent box (made up of the same glass as that of the chute panes). We mixed it carefully to avoid segregation. Each sample was stirred and filmed several times under the same lighting conditions as the chute; we repeated the procedure until we obtained 20 different images of the same sample. We averaged the gray value over the 20 images. We then derived a nonlinear curve that mapped the gray level $\theta$ to the concentration in the small beads $\phi^{s}: \phi^{s}=\theta^{3} / 9346-\theta^{2} / 266+0.614743 \theta+14.81$.

Stitching several images taken with a wide-angle lens (implying substantial image distortion, in particular on the image borders) is not easy. The overlapping field between two neighboring images was thin and distorted, which implied that images must be processed to correct distortion. After testing different solutions, we finally decided to correct the image perspective by imposing that the bed and free surface (which appeared as curved lines on raw images) must be straight lines. Images were also aligned so that there was no mismatch between the position of the bed and free surface from one image to another.

The velocity profiles were measured using classic particle imaging velocimetry techniques. ${ }^{30}$ Consistency was checked by visual inspection of different couples of images (brightness and flare might have biased velocity measurements).

\section{EXPERIMENTAL RESULTS}

\section{A. Preliminary tests}

Preliminary tests were carried out to delineate the flow rates (by varying the inflow flow-depth $h_{1}$ and $h_{2}$ ) and chute inclination $\zeta$ for which a steady uniform depth flow occurred (i.e., a flow for which the flow depth was uniform and constant over a significant length and for sufficiently long times). It turned out that our results were greatly influenced by minute changes in the chute inclination and the inflow rates, a situation that is encountered with monosized particles $^{31}$ and is exacerbated with binary mixtures. To illustrate this dependence, we report the results for a chute inclination of $\zeta=29^{\circ}$. Table I shows the flow depths at different positions along the flume as a function of the inflow conditions fixed by $h_{1}$ and $h_{2}$. The inflow depth $h_{1}$ (controlling the large-bead supply) could range from 0 to $60 \mathrm{~mm}$, but in practice, we had to narrow the $h_{1}$ range to $25-40 \mathrm{~mm}$ for the following reasons. For $h_{1}<25 \mathrm{~mm}$, the free surface was blurred by saltating particles; flows were then in a dilute kinetic regime, for which the theory outlined in Sec. II does not hold. For $h_{1}>40 \mathrm{~mm}$, a stationary layer made up of large beads and similar to the superstable heap reported by Taberlet et $_{\text {al. }}{ }^{32}$ formed along the base of the flume. For each $h_{1}$ value, $h_{2}$ values (controlling the small-bead supply) were increased from 6 to $14 \mathrm{~mm}$ in $2 \mathrm{~mm}$ steps. For $h_{2}<6 \mathrm{~mm}$, no steady inflow took place over the flume length monitored by the cameras (presumingly because the flume bottom was not rough enough for a mixture rich in large particles), while for $h_{2}>14 \mathrm{~mm}$, the large-bead flow rate was too low compared to the small-bead flow rate.

Table I shows that the large-bead flow rate depended on that of the smaller beads: for a given aperture of $h_{1}, Q_{\text {large }}$ decreased nearly linearly with increasing $h_{2}$. In contrast, when $h_{2}$ was fixed and $h_{1}$ varied, there was no clear trend for the total mass flow rate, which could decrease or increase with $h_{1}$.

No genuinely steady uniform depth region was observed in our flume, that is, the flow depth never reached a constant value, but slowly decreased along the chute. However, for some runs, this decrease was sufficiently slow for a nearly steady uniform flow to take place. For instance, for runs 4, 9, 10,12 , and 13, the difference in the flow depths between $x=50$ and $x=160 \mathrm{~cm}$ was lower than $7 \%$. For other runs, the 
TABLE II. Inflow flow-depth $h_{1}$ and $h_{2}$ in millimeters (see Fig. 2); flowdepth $h$ (in millimeters) of the steady uniform flow; mass flow rates in large and small particles, $Q_{\text {large }}$ and $Q_{\text {small }}$, respectively, in g/s; flow-depth averaged velocity $\bar{u}$ in $\mathrm{mm} / \mathrm{s}$. $h, Q_{\text {large }}, Q_{\text {small }}$, and $\bar{u}$ were measured in the steady uniform regions. Chute inclination $\zeta=29^{\circ}$.

\begin{tabular}{lcccccc}
\hline \hline Run & $h_{1}$ & $h_{2}$ & $h$ & $Q_{\text {large }}$ & $Q_{\text {small }}$ & $\bar{u}$ \\
\hline 22 & 24 & 9.5 & 24 & 35.7 & 47.2 & 34.5 \\
23 & 35 & 9.3 & 28 & 45.4 & 42.1 & 26.6 \\
24 & 20 & 6.3 & 19 & 34.7 & 23.6 & 35.2 \\
25 & 18.3 & 10.7 & 20 & 31 & 46 & 36.4 \\
\hline \hline
\end{tabular}

difference was close to $15 \%$, but on some occasions (e.g., run 16), it could be as high as $40 \%$. Flow-depth decrease implies that the flow was slightly accelerating, which may be the consequence of the roughness conditions imposed. Indeed, since the chute roughness was made up of small particles, the largest particles, which slid along this roughness when entering the chute, encountered weak resistance and accelerated vigorously. Note that for low $h_{1}$ values (i.e., for low flow rates in large beads), the flow-depth profile exhibited a complex pattern (e.g., see runs 2-5): the flow depth first decreased, then increased slowly toward a nearly constant value, and finally decreased again near the flume outlet. The flow-depth increase can be explained by the segregation process: the basal layer getting richer in small particles, the increase in the flow resistance caused the flow to decelerate. The decrease at the flume exit is common for slow, subcritical flows: when particles fall off the flume, the downstream normal stress decreases abruptly, which causes the flow to accelerate upstream (in subcritical flows, the disturbances can move upstream).

\section{B. Comparison with theory}

Gray and Chugunov's theory applies to a wide range of flow regimes, but since the segregation parameters $q$ and $D$ are neither known nor easy to measure independently, we focused our attention on steady uniform depth flows, which allowed us to use well-defined constraints when fitting these parameters to our data. After delineating the flow regimes, we then conducted additional tests to investigate the remixing and segregation in steady uniform depth flows. Table II reports the flow conditions for these specific runs. To show here how theory compares with experimental data, we have selected one typical run from these experiments, which will be analyzed in detail: run 22. For other runs, we will just provide the values of the fitted parameters (see Table III), the flow pattern being similar to that of run 22 .

To compare our data with theory, we first needed to determine the percolation rate $q$ and diffusivity $D$. To that end, we considered that very far downstream from the chute inlet, the particles size distribution was in the steady laterally uniform state, so that we could use the exact solution (11) to fit $q / D$ to a concentration profile. Figure 4 shows the steady laterally uniform concentration profile $\phi_{\text {uniform }}(z)$ measured at $x=70 \mathrm{~cm}$ from the inlet and the best fit obtained by adjusting Eq. (11) using a least-squares technique. The theoretical profile has an $S$-shape that closely describes the varia-
TABLE III. Values of the parameters for runs 22-25: parameter $\lambda$ of the velocity profile (20); diffusivity $D$ in $\mathrm{mm}^{2} \mathrm{~s}^{-1}$; percolation rate $q$ in $\mathrm{mm} \mathrm{s}^{-1}$; mean small-particle concentration $\bar{\phi}$; Péclet number $\mathrm{Pe}=q h / D$; depthaveraged shear rate $\bar{\gamma}=\bar{u} / h$ in s $^{-1}$. Chute inclination $\zeta=29^{\circ}$.

\begin{tabular}{ccccccc}
\hline \hline Run & $\lambda$ & $D$ & $q$ & $\bar{\phi}$ & $\mathrm{Pe}$ & $\bar{\gamma}$ \\
\hline 22 & 3.24 & 2.52 & 1.99 & 0.74 & 19 & 1.44 \\
23 & 3.1 & 2.08 & 1.23 & 0.50 & 16.5 & 0.95 \\
24 & 2.3 & 2.79 & 1.61 & 0.52 & 11 & 1.85 \\
25 & 2.9 & 2.66 & 1.74 & 0.67 & 13 & 1.81 \\
\hline \hline
\end{tabular}

tions in $\phi_{\text {uniform }}$ with depth, for parameters $\bar{\phi}=0.74$ and $\mathrm{Pe}=19$, i.e., $q h / D=19$. To show how sensitive the results are to changes in the Pe value, we have also plotted the theoretical curves corresponding to $\mathrm{Pe}=15$ and $\mathrm{Pe}=23$ (i.e., a variation of $\pm 20 \%$ with respect to the adjusted value $\mathrm{Pe}=19$, which corresponds to the magnitude of the uncertainty on $q$ and $D$ ) on the same plot.

The inflow condition (14) and the steady-state solution (11) are related by the conservation property (17). The downstream velocity $u$ plays a very important role in ensuring that the depth-averaged concentration $\bar{\phi}$ is correct far downstream. We assumed that $u$ was independent of $x$, but was dependent on $z$. Assuming a linear velocity profile with depth $^{18,21,24}$ significantly underpredicts the depth-averaged concentration $\bar{\phi}$ far downstream. Instead, we found a good approximation by fitting an exponential profile to experimental data. The data used for this were extracted from a $35 \mathrm{~cm}$ long window, where a nearly steady uniform flow occurred. We used the following parametrization:

$$
\frac{u(z)}{\bar{u}}=\frac{\lambda}{e^{\lambda}-1} \exp \left(\lambda \frac{z}{h}\right),
$$

where $\lambda$ is an adjustable parameter and $\bar{u}$ is the depthaveraged downstream velocity. There is no specific physical justification for this parametrization; it is just a convenient mathematical representation of concave velocity profiles.

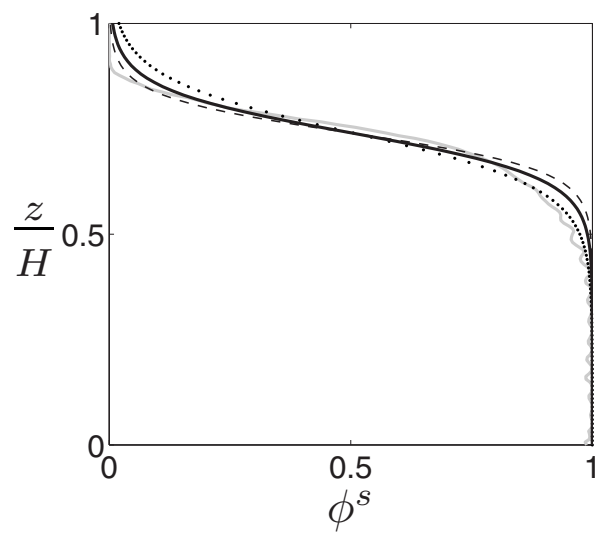

FIG. 4. Concentration profile measured at $x=70 \mathrm{~cm}$ from inlet (gray solid line) for run 22; the black solid line represents the theoretical concentration profile given by Eq. (11) with $\mathrm{Pe}=q h / D=19$ and $\bar{\phi}=0.74$. Data scaled with $H=h=24 \mathrm{~mm}$. To show how sensitive the curves are when altering the value of the Péclet number, we also plot the concentration profiles for $\mathrm{Pe}=15$ (dotted curve) and $\mathrm{Pe}=23$ (dashed curve). 

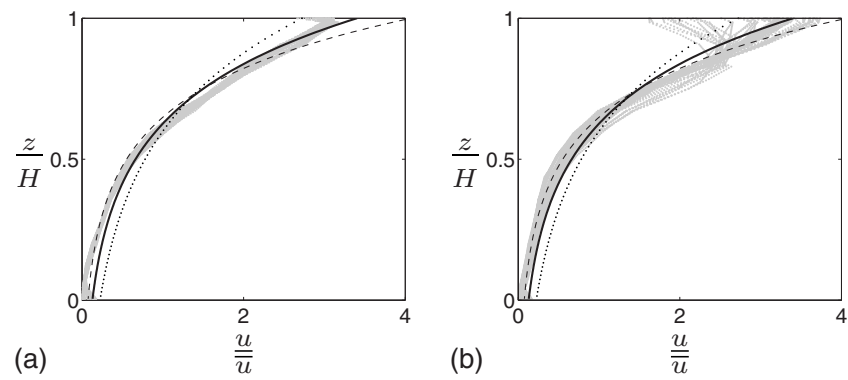

FIG. 5. Experimental (gray dots) and empirical (solid line) streamwise velocity profiles $u(x, z)$ for run 22. (a) Profiles taken in the steady uniform region (over a $35 \mathrm{~cm}$ long window from $x=35$ to $x=70 \mathrm{~cm}$ ); profiles were taken every $5.8 \mathrm{~mm}$. (b) Profiles in the upstream part of the flow (mixing region), where the velocity profile exhibits an $x$-dependence (over a $35 \mathrm{~cm}$ long window from $x=0$ to $x=35 \mathrm{~cm}$ ); profiles were taken every $5.8 \mathrm{~mm}$. The empirical profile (solid line) is obtained by fitting Eq. (20) on the data pertaining to the steady uniform region; we obtained $\lambda=3.24$. To give an idea of how sensitive the results are to changes in the $\lambda$ value, we report the curve corresponding to $\lambda=2.5$ (dotted line) and $\lambda=4.0$ (dashed line).

Figure 5 shows both the empirical profile $u(x, z)$ and the data. A reasonably good fit was obtained by taking $\lambda=3.24$. The data scattering around this fitted curve shows that the velocity profile was not completely independent of $x$, but the deviations from the mean trend were not significant, which justifies that we considered $u$ as a function of $z$ solely. In contrast, if we consider the mixing (i.e., the region in which the inverse grading process occurs) by taking the $35 \mathrm{~cm}$ window just upstream of this one, the resulting velocity was markedly different [see Fig. 5(b)]: clearly, there was a dependence on $x$, reflected here by a larger scattering of measurement points, and the top layer close to the free surface (approximately $20 \%$ of the total flow depth) experienced substantial acceleration. Figure 6 shows the normal velocity profiles $w(x, z)$ with the same conditions as for Fig. 5. In the steady uniform region [see Fig. 6(a)], the normal velocity is close to zero, but a recirculation cell is conspicuous in the upper half of the flow, where some velocity profiles were negative close to the free surface (which means that particles went down) while in the middle and above (for $z / h$ in the 0.4-0.8 range), all velocities were positive (particles went up). This recirculation cell in the uniform flow regions may be an indication that convection rolls disturbed the main flow (simple shear) or that the remixing caused recirculation. In the mixing region [see Fig. 6(b)], the velocity profiles exhibited much more variability, which was expected since the
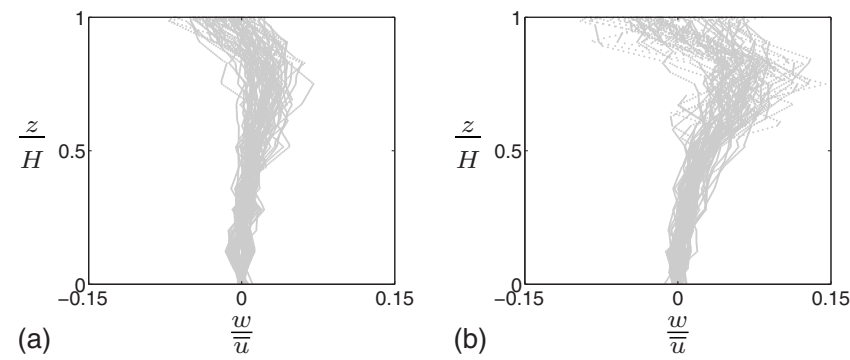

FIG. 6. Experimental normal velocity profiles $w(x, z)$ for run 22. (a) Profiles taken in the steady uniform region. (b) Profiles in the upstream part of the flow (mixing region). Same caption as for Fig. 5.
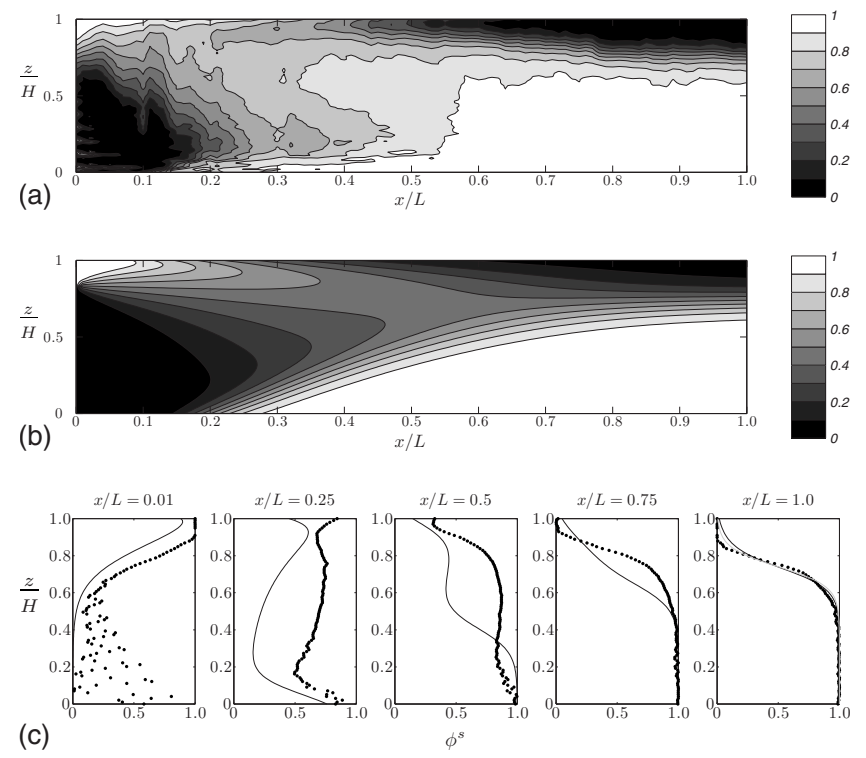

FIG. 7. Comparison between theory and experiment for run 22. (a) Concentration map for run 22. (b) Theoretical prediction with $D=2.52 \mathrm{~mm}^{2} / \mathrm{s}$, $\lambda=3.24$, and $q=1.99 \mathrm{~mm} / \mathrm{s}$. (c) Comparison between theoretical concentration profiles (solid lines) and data (dots) at a series of downstream locations. Data scaled with $H=h=24 \mathrm{~mm}$ (flow depth) and $L=0.7 \mathrm{~m}$ (length of the observing window). Note that the height to length ratio is 0.0343 , so that horizontal gradients are much less than they might appear.

finest particles at top went down while the largest particles in the middle of the flow went up.

Having determined an approximation for the nonlinear velocity field $u$ and the Péclet number Pe, we numerically solved Eq. (8) under steady uniform depth flow conditions, subject to the boundary conditions (10) and the inflow condition (14), using the pdepe routine of MATLAB. For this case, the governing Eq. (8) reduces to a nonlinear parabolic equation of the form

$$
\frac{\partial}{\partial x}(\phi u)-\frac{\partial}{\partial z}[q \phi(1-\phi)]=\frac{\partial}{\partial z}\left(D \frac{\partial \phi}{\partial z}\right) .
$$

In this equation, $D$ was crudely estimated so that agreement between theory and experiment looked correct; in practice, we tuned $D$ so that the computed $\phi=0.1$ contour line, which was obtained by solving Eq. (21) numerically, approached the experimental values. Although this procedure may seem somewhat cursory, it was not more arbitrary or inaccurate than other strategies we tested in parallel. Uncertainties on $D$ and $q$ could be as large as $\pm 20 \%$.

Figure 7 reports the variation in the concentration $\phi$ as a function of $x$ and $z$. Panel (a) reports the concentration map deduced from the raw image shown in Fig. 3; only the contour lines corresponding to a given level of concentration are represented. Plot (b) shows the concentration map obtained by solving Eq. (21) numerically. The lower plot gives a few concentration profiles measured at different places along the chute; we have also reported the theoretical predictions. Although the theoretical concentration map is consistent with the experimental map, there are a few differences in the mixing region. As shown in Fig. 7(c), the concentration profiles at the entrance and exit of the observing window had an 


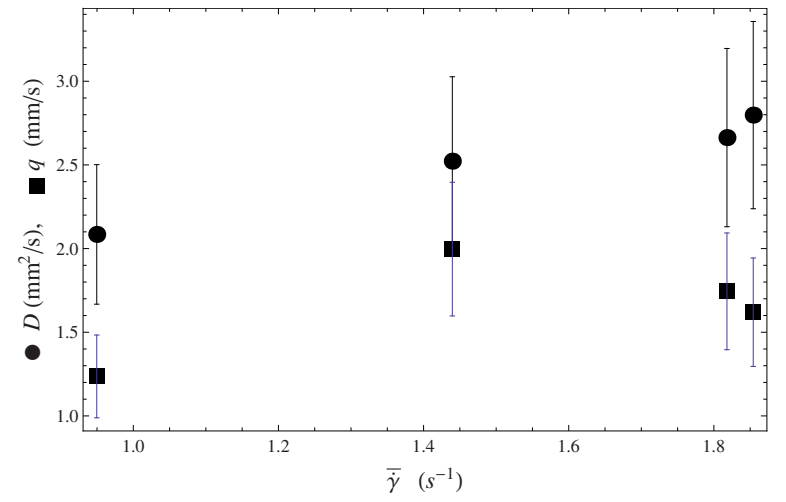

FIG. 8. (Color online) Variation in $q$ and $D$ with $\overline{\dot{\gamma}}$. Error bars with magnitude $\pm 20 \%$ are also reported.

$S$-shape, which is correctly accounted for by theory, but in the mixing region, the measured profiles were blunt whereas theory predicts a more contrasted profile between the top and the bottom layers. Note also that for the leftmost subplot in Fig. 7(c) (for $x / L=0.01$ ), the scatter in the measured concentration $\phi^{s}$ reflects the layering effect discussed above.

Several explanations can be put forward to explain the discrepancies in the mixing region, where the percolation took place. Some authors including Schröter et $a l^{33}$ and Ulrich et $a l .{ }^{34}$ pointed out that friction at the sidewall may induce convection rolls and promote remixing rather than segregation. Although this phenomenon was mainly observed in vertically shaken mixtures, we can think that a similar process occurs in gravity-driven shear flows in narrow chutes: indeed, sidewall friction induces a strong velocity gradient in the cross-stream direction, which drives the smaller particles laterally to the sidewall and initiates a secondary flow; ${ }^{35,36}$ note that the packing effect on the wall (for binary mixtures, the finest particles are more easily found next to a flat surface than the coarsest one) can also explain this phenomenon. Taking a closer look at the free surface revealed that small particles came out in regularly spaced groups of a few particles all along the centerline, which suggested the existence of convection rolls with a well-defined length. Another explanation for the discrepancies lies in the difference between the actual velocity field in the mixing region and that used in the computation. Recall that the velocity profile (20) used in the computation was fitted to data obtained from a $35 \mathrm{~cm}$ long window downstream of the mixing region (where a steady uniform flow region took place). As shown in Fig. 5(b), the velocity profile in the mixing region differed from the assumed profile near the free surface. At the same time, the normal velocity component $w$ was nonzero (see Fig. 6), which confirms that the velocity field in the mixing region was not the fully developed field considered in the numerical computations.

We repeated the procedure for other flow conditions. Table III reports the best-fit values of the velocity-profile parameter $\lambda$, the diffusivity $D$, the percolation rate $q$, the mean small-particle concentration $\bar{\phi}$, and the Péclet number Pe. As shown by Table III, while there is little dependence of the segregation parameters $q$ and $D$ on the velocity parameter $\lambda$, the segregation parameters are found to slightly de- pend on the depth-averaged shear rate $\bar{\gamma}=\bar{u} / h$ : the diffusivity seems to linearly increase with $\overline{\dot{\gamma}}$, while the percolation rate curve is bell-shaped with a maximum reached at $\overline{\dot{\gamma}}=1.4 \mathrm{~s}^{-1}$. This contrasts with early observations by Bridgwater et al., ${ }^{22}$ who found that the percolation rate $q$ is proportional to the shear rate. The variation range of $\lambda$ is however too narrow to be conclusive on this point. Furthermore, given the uncertainty on the $q$ and $D$ and the narrow range of variation in $\overline{\dot{\gamma}}$, it is difficult to be conclusive on the dependence of $q$ and $D$ on $\overline{\dot{\gamma}}$ (Fig. 8).

\section{SUMMARY AND CONCLUDING REMARKS}

We used a narrow flume to study the evolution of a binary granular mixture made up of spherical glass beads ( 1 and $2 \mathrm{~mm}$ in diameter). Initially, the mixture was normally graded, with all the small particles on top of the coarse grains. When flowing, particles segregated, that is, the smallest particles percolated to the bottom, while the largest were squeezed up toward the free surface. As usual for this beadsize ratio, a competing process, here referred to as diffusive mixing, smeared out the jump from the small-particle to the large-particle layers. Using a high-speed camera, we measured the evolution of the small-particle concentration as a function of time and position (together with the velocity profile) at the sidewall.

The experimental data were then used to test a theoretical model that describes segregation-remixing in dry granular avalanches as a nonlinear advection diffusion process. ${ }^{14}$ This equation involves two parameters: the maximum percolation rate $q$ (which controls the segregation strength) and diffusivity $D$ (which quantifies the diffusive effects). Using a steady laterally uniform solution, which specifies the smallparticle distribution under steady uniform depth conditions, we fitted the ratio $q / D$, while the parameter $D$ was tuned qualitatively until a visually good agreement was obtained between theory and experiment in the mixing regime (the region in which the grading inversion occurred). Excellent agreement between theory and experiment was obtained in the downstream region, in which the concentration profile reached an equilibrium state and exhibited an $S$-shape. However, to get the right depth-averaged concentration $\bar{\phi}$ far downstream, it was very important to use the measured velocity profiles. Upstream, in the mixing region, theoretical profiles did not closely match the experimental data. One reason for this discrepancy lies in the velocity distribution: in the theoretical applications, we assumed that the velocity profile depended on depth solely (i.e., it showed no dependence on the downstream position). Experimentally, we observed that this assumption held true sufficiently far downstream of the flume inlet, but in the mixing region, which was located near the chute entrance, the velocity field was quite complicated. Another possible explanation lies in the packing effects near the wall: small particles are more likely to concentrate near the sidewall in the mixing region than in regions dominated by pure (graded or inversely graded) states. This is consistent with what Golick and Daniels ${ }^{11}$ reported (note that their measurements may have been influ- 
enced by centrifugal effects) and can be explained by a cross-stream (in the $y$-direction) particle segregation. This also explains why this effect dies down when the particles separate out. A third way of explaining the discrepancy could be that the flux function in Eq. (8) proposed by Gray and Chugunov $^{14}$ is not the quadratic form $-q \phi(1-\phi)$, but may be better described by a different convex curve. For instance, expressions such as $f^{s}=\phi^{s}-q\left(\phi^{s} \phi^{l}\right)^{n}$ and $f^{l}=\phi^{l}+q\left(\phi^{s} \phi^{l}\right)^{n}$ (with $n<1$ ) satisfy the constraints $f^{s}+f^{l}=1$ and $f^{\nu}=1$ when $\phi^{\nu}=1(\nu=l, s)$, and lead to a nonquadratic convex flux function $-q[\phi(1-\phi)]^{n}$ in Eq. (8). More work is needed to elucidate the influence of the flux function on the segregationremixing process.

Several experiments were conducted at constant slope, but with varying inflow rates. These experiments showed that the model parameters $q$ and $D$ did not depend on the shear-rate strength $\lambda$, but may slightly depend on the depthaveraged shear rate $\overline{\dot{\gamma}}$, a finding that contrasts with earlier observations made by Bridgwater et al., ${ }^{22}$ Golick and Daniels, ${ }^{11}$ and May et al., ${ }^{12}$ who reported significant variations in $q$ with shear rate. This suggests that the segregation and diffusive remixing are only weakly dependent on the depth-averaged shear rate $\overline{\dot{\gamma}}$ once sufficient dilation has occurred. The variation in $\bar{\gamma}$ is too narrow to be conclusive at this point.

\section{ACKNOWLEDGMENTS}

C.A. is grateful for the financial support provided by the Swiss National Science Foundation under Grant No. 200021105193, the competence center in Mobile Information and Communication Systems (a center supported by the Swiss National Science Foundation under Grant No. 5005-67322, MICS project), and the competence center in Environmental Sciences (TRAMM project). Part of this research was performed during three research visits to the Hydraulics Laboratory at EPFL Lausanne and was supported by generous funding from the School of Architecture, Civil and Environmental Engineering, as well as ERCOFTAC (European Research Community on Flow, Turbulence and Combustion). N.G. also acknowledges the support from the NERC, Grant Nos. NER/A/S/2003/00439 and NE/E003206/1, as well as an EPSRC Advanced Research Fellowship, Grant Nos. GR/ S50052/01 and GR/S50069/01.

${ }^{1}$ P. Bartelt and B. McArdell, "Granulometric investigations of snow avalanches," J. Glaciol. 55, 829 (2009).

${ }^{2}$ V. Jomelli and P. Bertran, "Wet snow avalanche deposits in the French Alps: Structure and sedimentology," Geogr. Ann., Ser. A: Phys. Geogr. 83, 15 (2001).

${ }^{3}$ M. Ramaioli, "Granular flow simulations and experiments for the food industry," Ph.D. thesis, EPFL, 2007.

${ }^{4}$ S. Savage and C. Lun, "Particle size segregation in inclined chute flow of dry cohesionless granular solids," J. Fluid Mech. 189, 311 (1988).

${ }^{5}$ E. Linares-Guerrero, C. Goujon, and R. Zenit, "Increased mobility of bidisperse granular avalanches," J. Fluid Mech. 593, 475 (2007).

${ }^{6} \mathrm{~J}$. Gray, "Particle size segregation in granular avalanches: A brief review of recent progress," in IUTAM-ISIMM Symposium on Mathematical Modeling and Physical Instances of Granular Flows, edited by J. Goddard, J. Jenkins, and P. Giovine (AIP, Melville, NY, 2010), Vol. 1227, pp. 343362.

${ }^{7}$ J. Gray and K. Hutter, "Pattern formation in granular avalanches," Continuum Mech. Thermodyn. 9, 341 (1997).
${ }^{8}$ J. M. Ottino and D. V. Khakhar, "Mixing and segregation of granular materials," Annu. Rev. Fluid Mech. 32, 55 (2000).

${ }^{9}$ A. Kudrolli, "Size separation in vibrated granular matter," Rep. Prog. Phys. 67, 209 (2004).

${ }^{10} \mathrm{~V}$. Dolgunin and A. Ukolov, "Segregation modeling of particle rapid gravity flow," Powder Technol. 83, 95 (1995).

${ }^{11}$ L. Golick and K. Daniels, "Mixing and segregation rates in sheared granular materials," Phys. Rev. E 80, 042301 (2009).

${ }^{12}$ L. May, L. Golick, K. Phillips, M. Shearer, and K. Daniels, "Shear-driven size segregation of granular materials: Modeling and experiment," Phys. Rev. E 81, 051301 (2010).

${ }^{13}$ Y. Srebro and D. Levine, "Role of friction in compaction and segregation of granular materials," Phys. Rev. E 68, 061301 (2003).

${ }^{14}$ J. Gray and V. Chugunov, "Particle-size segregation and diffusive remixing in shallow granular avalanches," J. Fluid Mech. 569, 365 (2006).

${ }^{15} \mathrm{~S}$. Savage and K. Hutter, "The motion of a finite mass of granular material down a rough incline," J. Fluid Mech. 199, 177 (1989).

${ }^{16}$ J. M. N. T. Gray, Y. C. Tai, and S. Noelle, "Shock waves, dead-zones and particle-free regions in rapid granular free-surface flows," J. Fluid Mech. 491, 161 (2003).

${ }^{17}$ Y. Forterre and O. Pouliquen, "Flows of dense granular media," Annu. Rev. Fluid Mech. 40, 1 (2008).

${ }^{18}$ A. Thornton, J. Gray, and A. Hogg, "A three-phase mixture theory for particle size segregation in shallow granular free-surface flows," J. Fluid Mech. 550, 1 (2006).

${ }^{19}$ L. Morland, "Flow of viscous fluids through a porous deformable matrix," Surv. Geophys. 13, 209 (1992).

${ }^{20}$ D. Drew and S. Passman, Theory of Multicomponent Fluids (Springer, New York, 1999).

${ }^{21}$ J. Gray and A. Thornton, "A theory for particle size segregation in shallow granular free-surface flows," Proc. R. Soc. London, Ser. A 461, 1447 (2005).

${ }^{22}$ J. Bridgwater, W. Foo, and D. Stephens, "Particle mixing and segregation in failure zones-Theory and experiment," Powder Technol. 41, 147 (1985).

${ }^{23}$ S. Sarkar and D. Kharkhar, "Experimental evidence for a description of granular segregation in terms of the effective temperature," Europhys. Lett. 83, 54004 (2008).

${ }^{24}$ J. M. N. T. Gray, M. Shearer, and A. R. Thornton, "Time-dependent solutions for particle-size segregation in shallow granular avalanches," Proc. R. Soc. London, Ser. A 462, 947 (2006).

${ }^{25}$ M. Shearer, J. M. N. T. Gray, and A. R. Thornton, "Stable solutions of a scalar conservation law for particle-size segregation in dense granular avalanches," Eur. J. Appl. Math. 19, 61 (2008).

${ }^{26}$ J. Gray and C. Ancey, "Particle size-segregation, recirculation, and deposition at coarse particle rich flow fronts," J. Fluid Mech. 629, 387 (2009).

${ }^{27}$ R. Skeel and M. Berzins, "A method for the spatial discretization of parabolic equations in one space variable," SIAM (Soc. Ind. Appl. Math.) J. Sci. Stat. Comput. 11, 1 (1990).

${ }^{28}$ J. M. N. T. Gray and B. P. Kokelaar, "Large particle segregation, transport and accumulation in granular free-surface flows," J. Fluid Mech. 652, 105 (2010).

${ }^{29}$ P. Jop, Y. Forterre, and O. Pouliquen, "Crucial role of side walls for granular surface flows: Consequences for the rheology," J. Fluid Mech. 541, 167 (2005).

${ }^{30} \mathrm{M}$. Raffel, C. Willert, S. Wereley, and J. Kompenhans, Particle Image Velocimetry (Springer, Berlin, 2007).

${ }^{31} \mathrm{C}$. Ancey, "Dry granular flow down an inclined channel: Experimental investigations on the frictional-collisional regime," Phys. Rev. E 65, 011304 (2001)

${ }^{32}$ N. Taberlet, P. Richard, A. Valance, W. Losert, J. M. Pasini, J. Jenkins, and R. Delannay, "Superstable granular heap in a thin channel," Phys. Rev. Lett. 91, 264301 (2003).

${ }^{33}$ M. Schröter, S. Ulrich, J. Kreft, J. Swift, and H. Swinney, "Mechanisms in the size segregation of a binary granular mixture," Phys. Rev. E 74, 011307 (2006).

${ }^{34}$ S. Ulrich, M. Schröter, and H. Swinney, "Influence of friction on granular segregation," Phys. Rev. E 76, 042301 (2007).

${ }^{35} \mathrm{R}$. Bagnold, "Experiments on a gravity-free dispersion of large solid spheres in a Newtonian fluid under shear," Proc. R. Soc. London 225, 49 (1954).

${ }^{36}$ D. Leighton and A. Acrivos, "The shear-induced migration of particles in concentrated suspensions," J. Fluid Mech. 181, 415 (1987). 